\title{
Medicamentos Fitoterápicos mais comercializados de 2020 a 2021 em uma drogaria situada em Manaus - AM - Brasil
}

\author{
Most marketed herbal medicines from 2020 to 2021 in a drugstore located in Manaus - AM - Brazil \\ Medicamentos a base de hierbas más comercializados de 2020 a 2021 en una farmacia situada en \\ Manaus - AM - Brasil
}

Recebido: 05/11/2021 | Revisado: 12/11/2021 | Aceito: 19/11/2021 | Publicado: 28/11/2021

\section{Resumo}

Geise Luciane Rocha Campos

ORCID: https://orcid.org/0000-0002-4901-2083 Centro Universitário do Norte, Brasil E-mail: geise2009@gmail.com

Luana Maria Ferreira do Nascimento ORCID: https://orcid.org/0000-0001-8093-891X Centro Universitário do Norte, Brasil E-mail: lumadona3089@gmail.com

Marcos Túlio da Silva

ORCID: https://orcid.org/0000-0002-7653-4944 Faculdade Uninorte, Brasil

E-mail: marcostulio39@gmail.com

Objetivos: Este artigo tem como principal objetivo analisar os fitoterápicos que foram mais vendidos entre setembro de 2020 e setembro de 2021 em uma drogaria da cidade. Métodos: listar quais os principais medicamentos fitoterápicos vendidos entre os meses de setembro de 2020 e setembro de 2021; pontuar os efeitos do uso irracional do medicamento fitoterápico a base de Mikania glomerata (conhecida popularmente como guaco); este trabalho trata-se de uma pesquisa exploratória realizada, utilizando pesquisa documental e bibliográfica. Resultados: foram vendidos 6620 fitoterápicos entre setembro/2020 a setembro/2021, dentre esse total foram vendidos 1820 medicamentos fitoterápicos e o mais vendido foi o medicamento fitoterápico com a espécie vegetal Mikania glomerata, com 384 unidades vendidas. Conclusão: a quantidade de procura relacionado ao xarope Guaco está relacionada com a eficiência desse fitoterápico atrelada a alternativa de medicamentos em meio a pandemia do SARS-CoV-2, que faz com que o indivíduo apresente sintomas gripais e procure essas medicações.

Palavras-chave: Fitoterápicos; Mikania glomerata; Comercialização.

\begin{abstract}
Objectives: This article has as its main objective to analyze the phytotherapy drugs that were most sold between September 2020 and September 2021 in a drugstore in the city. Methods: To list the main phytotherapeutic drugs sold between September 2020 and September 2021; punctuate the effects of irrational use of herbal medicine based on Mikania glomerata (popularly known as guaco); this work is exploratory research carried out, using documentary and bibliographic research. Results: 6620 herbal medicines were sold between September/2020 and September/2021, out of this total, 1820 herbal medicines were sold and the most sold was the herbal medicine with the plant species Mikania glomerata, with 384 units sold. Conclusion: the amount of demand related to Guaco syrup is related to the efficiency of this phytotherapy linked to the alternative of medicines during the SARS-CoV-2 pandemic, which causes the individual to present flu symptoms and seek these medications.
\end{abstract}

Keywords: Phytotherapeutics; Mikania glomerata; Commercialization.

\section{Resumen}

Objetivos: Este artículo tiene como objetivo principal analizar los medicamentos a base de hierbas que más se vendieron entre septiembre de 2020 y septiembre de 2021 en una farmacia de la ciudad. Métodos: enumerar los principales medicamentos fitoterapéuticos vendidos entre los meses de septiembre de 2020 y septiembre de 2021; puntualizar los efectos del uso irracional de la medicina herbal basada en Mikania glomerata (conocida popularmente como guaco); este trabajo es una investigación exploratoria realizada, utilizando investigación documental y bibliográfica. Resultados: Entre septiembre / 2020 y septiembre / 2021 se vendieron 6620 medicinas herbales, de este total se vendieron 1820 medicinas herbales y la más vendida fue la herbolaria con la especie vegetal Mikania glomerata, con 384 unidades vendidas. Conclusión: la cantidad de demanda de jarabe de Guaco está relacionada con la eficacia de este fitoterápico vinculado a la alternativa de medicamentos en medio de la pandemia de SARS-CoV-2, que hace que el individuo presente síntomas de gripe y busque estos medicamentos.

Palabras clave: Fitoterápicos; Mikania glomerata; Comercialización. 


\section{Introdução}

A fitoterapia é conhecida como uma prática arcaica e milenar. Desde os primórdios da origem humana, as plantas medicinais se tornaram alternativas para cura e tratamento de diversas doenças, alívio de dor e cura de patologias. Esses conhecimentos foram sendo repassados de uma geração à outra através do senso comum, principalmente pelo seu uso ser motivado através de razões sociais ou econômicas, como dificuldades ao acesso à uma consulta médica ou ainda, na aquisição de medicamentos farmacêuticos, devido à localização, como no caso de municípios localizados em áreas de difícil acesso à drogarias, como a zona rural (Dantas,2019).

Dessa maneira, o uso de plantas medicinais é realizado por povos antigo que se utilizavam de raízes e plantas para tratamento de doenças e rituais de cura. Contudo, somente nos últimos anos que os estudos foram se avançando para a verificação da eficácia relacionada com as substâncias ativas pertencentes à propriedade, comprovando-se os mecanismos de ação e a efetividade dos compostos ativos dessas plantas em ação com o corpo humano (Meotti et al., 2020).

Em relação ao Brasil, os estudos e práticas relacionadas ao uso de medicamentos fitoterápicos estão em constante desenvolvimento, presente em variadas regiões do país com a implantação de programas destinados para explorar mais as propriedades dessas plantas com o intuito de verificar a sua eficácia (Ibiapina et al., 2019).

No Amazonas, a biodiversidade relacionada a flora presente em toda a região permite com que as plantas medicinais sejam utilizadas de forma empírica, com indicações realizadas e embasadas na cultura local. Em contrapartida, essas indicações estão sendo evidenciadas por estudos científicos e tecnológicos com finalidade de criar novas oportunidades ou alternativas voltadas para o uso de plantas para uso terapêutico ou também para fins ambulatoriais (Evangelista et al., 2013).

Assim, o uso de plantas medicinais para a produção dos medicamentos fitoterápicos advém do interesse no tratamento de patologias, por isso cada vez mais novos estudos estão buscando novas tecnologias para utilizar plantas medicinais e suas propriedades para auferir resultados e reações que possam contribuir com a redução de efeitos patológicos de algumas doenças (Haraguchi et al., 2020).

Com alguns estudos sendo realizados, alguns medicamentos fitoterápicos puderam ser utilizados como uma medicina alternativa para algumas patologias, como o caso dos medicamentos fitoterápicos a base de Mikania glomerata Sprengel, pertencente aos Asteraceae, conhecida de forma popular como Guaco, possuidora de propriedades específicas para sintomas de asmas, bronquite e no combate da tosse. Outros estudos mais aprofundados evidenciaram que Guaco também pode apresentar resultados eficientes no tratamento de alergias, sendo antimicrobiano, analgésico, anti-inflamatório, antioxidante e antidiarreico (Silva, 2019).

Com isso, estudos validam o seu uso alternativo relacionado à eficácia voltada para alguns sintomas ou patologias comprovadas por ensaios farmacológicos, pré-clínicos e clínicos de que os medicamentos fitoterápicos podem apresentar eficácia semelhante ou maior contra determinadas patologias se comparado com outros medicamentos atuantes a determinadas enfermidades (Araújo; Carolli; Alves,2020).

Outros estudos apresentados evidenciam a eficácia de medicamentos fitoterápicos para o tratamento de osteoporose, como a isoflavona, indicada principalmente para mulheres mais jovens, bem como fitoterápicos a base de cúrcuma que apresenta ação contra a deterioração da estrutura óssea diminuindo com isso a esteoclastogênese (Esteves et al., 2020).

A procura por medicamentos fitoterápicos também é considerável por causa do seu preço, comparando-se outros medicamentos apresenta um valor menor e isso pode conduzir a uma grande procura, principalmente pela ideia de que se trata de substâncias ativas naturais e que pode não ter contraindicações (Fernandes, 2020).

Este artigo tem como principal objetivo analisar os fitoterápicos que foram mais vendidos entre setembro de $2020 \mathrm{e}$ setembro de 2021 em uma drogaria da cidade. Busca-se listar quais os principais medicamentos fitoterápicos vendidos entre os meses de setembro de 2020 e setembro de 2021; pontuar os efeitos do uso irracional do guaco; evidenciar os estudos e seus 
respectivos resultados com o uso do xarope guaco.

Esse resultado também trouxe impactos para as demandas do uso de medicamentos fitoterápicos para dentro do Sistema Único de Saúde, através da criação de programas destinados para a implantação desses medicamentos para ser uma alternativa para todos os usuários, uma vez que há comprovações de que alguns fitoterápicos são eficientes para determinados sintomas (Ribeiro, 2019).

A principal problemática que envolve o uso desse medicamento fitoterápico é o uso irracional que envolve a compra desse medicamento, pois ao ser usado de forma irracional alguns efeitos podem ser evidenciados como problemas hemorrágicos. Perante a isto, pode-se surgir uma principal indagação: qual fitoterápico mais entre setembro de 2020 e setembro de 2021, em uma drogaria localizada na cidade de Manaus?

\section{Metodologia}

A pesquisa teve uma abordagem qualiquantitativa, refere-se a um estudo exploratório realizado em uma drogaria localizada na cidade de Manaus, utilizando-se como principais técnicas as análises documentais e observacionais. Tratando-se de uma pesquisa exploratória o estudo reúne informações essenciais sobre o objeto mapeado delimitando a pesquisa (Severino, p. 107, 2018). Logo a pesquisa exploratória tem objetivo de investigar tudo sobre um determinado objeto de maneira simples retratando a realidade buscando o máximo de conhecimento (Pereira, p. 33, 2018).

Dessa maneira, para análise documental foram realizados mapeamentos para verificar a quantidade de vendas dos fitoterápicos que são comercializados nessa drogaria. Para análise observacional, foram verificadas as principais causas que conduziram os clientes a comprarem os medicamentos fitoterápicos.

Para essa pesquisa foram adotados dois critérios, sendo inclusão e exclusão. Para a inclusão participaram da pesquisa somente os principais fitoterápicos de maior circulação de vendas entre os anos de 2021 e 2020 , e que puderam ser quantificados. Para o critério de exclusão, foram excluídos das pesquisas os outros medicamentos e informações de vendas de fitoterápicos anteriores ao ano de 2020.

Dessa maneira, após as coletas de dados foram submetidos a análise descritiva aonde todos os dados foram incluídos em planilhas eletrônicas, para que assim fosse possível verificar a distribuição entre os anos estipulados para que pudesse embasar o referencial teórico com os resultados obtidos.

\section{Resultados e Discussão}

De acordo com a pesquisa realizada no Sistema interno de vendas da drogaria estudada, verificou-se que no período pesquisado entre setembro de 2020 e setembro 2021, a drogaria vendeu 6620 medicamentos. Dessa quantidade, foram registrados 1820 fitoterápicos dos mais variados e para diversas finalidades terapêuticas. O fluxo desse mapeamento pode ser observado conforme Figura 1, abaixo: 
Figura 1 - Medicamentos fitoterápicos comercializados entre setembro de 2020 e setembro de 2021 conforme levantamento realizado em uma drogaria da cidade de Manaus

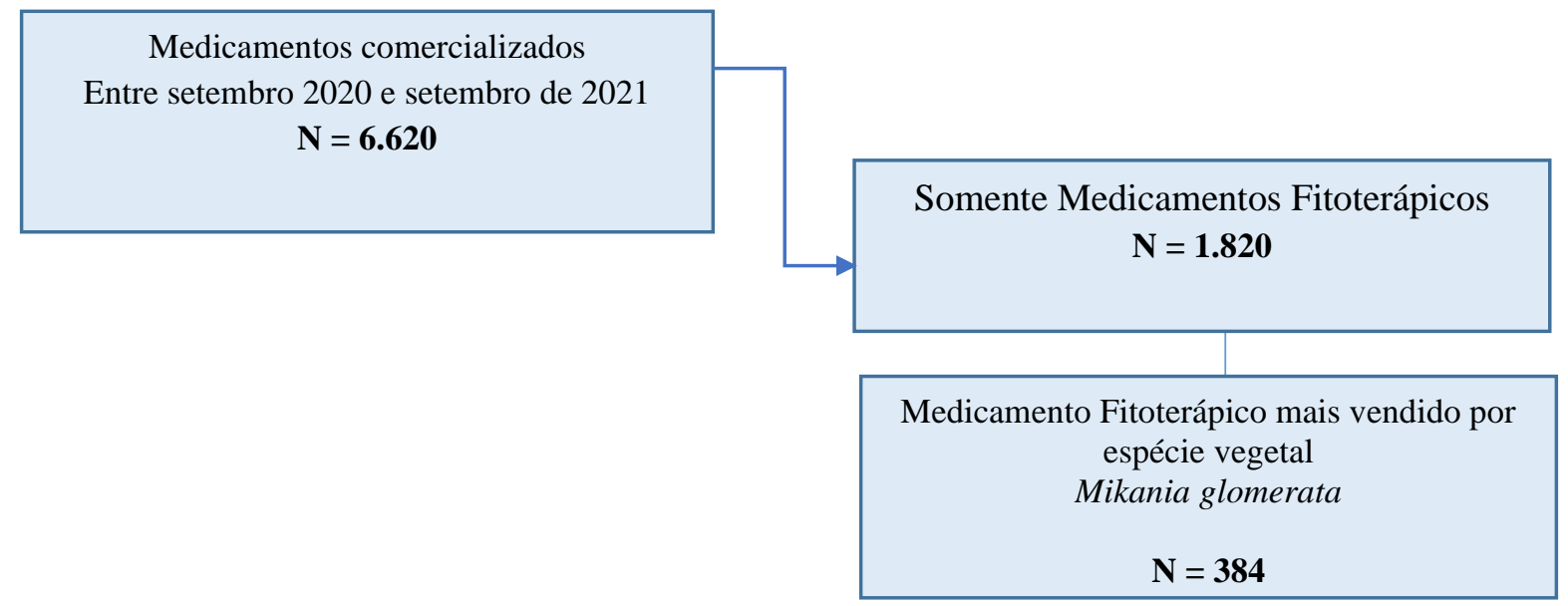

Fonte: Autores (2021).

Entre os fitoterápicos mais vendidos destaca-se o que contêm a espécie vegetal medicinal Mikania glomerata $(\mathrm{n}=384)$, Plassiflora incarnata $(\mathrm{n}=292)$, Lepidium meyenii $(\mathrm{n}=201)$. Abaixo temos a tabela 1com todos os medicamentos fitoterápicos mais vendidos por espécie vegetal medicinal entre entre setembro de 2020 e setembro de 2021

Tabela 1. Medicamentos fitoterápicos mais vendidos por espécie vegetal medicinal

\begin{tabular}{|c|c|}
\hline Espécie vegetal medicinal & Quantidade (unidades) \\
\hline Mikania glomerata & 384 \\
\hline Plassiflora incarnata & 292 \\
\hline Lepidium meyenii & 201 \\
\hline Senna alexandrina e Cassia fistula & 173 \\
\hline Hedera helix & 163 \\
\hline Valeriana officinalis & 127 \\
\hline Peumus boldus & 89 \\
\hline Rubra rosa & 76 \\
\hline Ananas comosus & 64 \\
\hline Plantago ovata & 56 \\
\hline Ginko biloba & 42 \\
\hline Cynara scolymus & 33 \\
\hline Isoflavona & 28 \\
\hline Rheum palmatum $L$. & 28 \\
\hline Silybum marianum & 21 \\
\hline Salix alba $L$. & 13 \\
\hline Pelargonium sidoides & 13 \\
\hline Aloe ferox + Gentiana lutea & 8 \\
\hline Symphytum officinale & 5 \\
\hline Aesculus hippocastanum $L$. & 4 \\
\hline Total & 1820 \\
\hline
\end{tabular}

Fonte: Autores (2021). 
Diante da pesquisa realizada o medicamento fitoterápico a base de Mikania glomerata (conhecido popularmente como Guaco) foi o mais comercializado entre o ano de 2020 e 2021 . Esse medicamento fitoterápico tem propriedades estão voltadas para o tratamento de doenças respiratórias, tosse, gripe e bronquite. Seu destaque para essa atuação se dá pelo fato de possuir propriedades analgésicas e anti-inflamatórias (Machado et al., 2018).

Contudo, sabe-se que a procura por um fitoterápico que contribuísse com o tratamento de gripe e doenças respiratórias foi também motivada pela iniciativa de proteção de diversas pessoas contra a gravidade do vírus Sars-Cov-2, mais conhecido como Coronavírus, pois os sintomas desse vírus se tornam mais impactantes para quem já tem um algum problema respiratório, isso porque o guaco apresenta prioridades antimicrobianas. (Diniz et al., 2021).

Em um estudo realizado por Silva et al., (2021) verificou-se o quanto o guaco ações antimicrobianas. No estudo foi feito uma avaliação através da Concentração Inibitória Mínima e Concentração Bactericida Mínima. No estudo o guaco apresentou atividade antimicrobiana para a bactéria gram-positiva Staphylococcus aureus. Fator esse relevante que pode ter contribuído para o aumento das vendas no período de 2020 a 2021 na drogaria estudada.

Em outro estudo parecido realizado por Amorim et al., (2020) evidenciou-se a ação direta do guaco causando broncodilatação e relaxamento da musculatura lisa respiratória, que está diretamente relacionada com o bloqueio dos canais de cálcio e com ações anti-inflamatória e antialérgica, sendo assim capaz de desobstruir as vias aéreas inflamadas.

Para Santos (2019) torna-se extremamente relevante realizar vendas de medicamentos que tenham bulas para justamente poder fornecer todas as informações necessárias para que os compradores possam compreender todos os efeitos que podem acontecer caso seja realizado o uso irracional do fitoterápico, principalmente no momento em que, há uso de forma irracional como no caso de esquecer de tomar o remédio no dia indicado ou na posologia indicada.

Por outro lado, Gomes e Pereira (2019), mesmo sendo um medicamento fitoterápico existem contraindicações que precisam ser levadas em consideração, como no caso de pessoas que estão em tratamento com medicamentos anti-inflamatório não esteroide (AINEs), isso porque a substâncias presentes nas folhas do guaco e que tem capacidade de interferir na coagulação sanguínea do indivíduo.

Dessa forma Ribeiro (2020) afirma que os efeitos do guaco corroboram para o aumento da procura por esse medicamento em tempo de pandemia, por causa de sintomas gripais. O guaco tem funções broncodilatadoras, antitussígeno e expectorante, relacionado com o tratamento de trato respiratório, referente a tosses persistentes por isso se torna um grande auxiliar para problemas respiratórios.

Nesse contexto, Ribeiro (2019), ao realizar uma pesquisa relacionada à eficácia de fitoterápicos e ao seu uso, evidenciou que Mikania Glomerata é uma planta medicinal que é registrada pela ANVISA e faz parte de programas voltados para a distribuição de fitoterápicos pelo Sistema Único de Saúde. Essa participação advém da eficiência dos seus resultados comprovados que atraem mais clientes para a compra desse medicamento.

Teófilo e Uhlmann (2021) trouxe no seu estudo a eficiência do xarope guaco para a eficiência nas "afecções pulmonares" onde possui o efeito broncodilatador, antitussígena, expectorante e edematogênica, onde os resultados para esses problemas foram evidenciados quando comprovado a redução dos problemas respiratórios devido a atuação da cumarina, onde para que possa ter esse efeito precisa ter uma dose diária de 0,5 a $5 \mathrm{mg}$ de cumarina.

Nos estudos de Silva e Teixeira (2019), foi testada a ação antimicrobiana do guaco onde foi feita a metodologia de micro-diluição em caldo, que é justamente uma técnica que envolve um grande número de bactérias. Contudo nesta pesquisa as folhas de Mikania glomerata não inibiram o crescimento das cepas microbianas provenientes na pesquisa, evidenciando a importância de estudos e testes para a indústria farmacêutica para a eficiência dos resultados de medicamentos fitoterápicos como o guaco. 
Outro estudo significante realizado por Marques et al., (2019), foram feitas comparações entre prescrições farmacêuticas entre medicamentos fitoterápicos, encontrou-se a ausência de prescrições nas vendas de fitoterápicos, por isso, a pesquisa evidencia a importância da atuação do farmacêutico para orientar todos os consumidores na hora de vender esses medicamentos para que todos possam ter conhecimento dos efeitos que possam aparecer durante o uso irracional.

\section{Considerações Finais}

O guaco (Mikania Glomerata) apresentou, na pesquisa, suas ações comprovadas para atuar contra tosse, sintomas gripais e problemas broncorespiratórios. Esses sintomas são semelhantes à atuação do vírus SarS-Cov-2, que atuou de forma mais intensa no ano de 2020 no Amazonas.

Com isso, a escassez de consultas médicas nesse período ou a suspensão de atendimentos médicos para prescrição de medicamentos que possam auxiliar pessoas com asma ou respiratórios fez com que esses clientes buscassem por guaco para reduzir os sintomas da tosse e gripe. Contudo, usar esses medicamentos sem a devida orientação os efeitos também podem ser contraditórios, como a presença de hemorragia, aumento de frequência cardíaca, hipertensão, dores de cabeça ou até mesmo prisão de ventre.

Assim, o resultado do levantamento realizado na drogaria espelhou esse momento pois foram realizadas 384 vendas do medicamento fitoterápico guaco entre os meses de setembro de 2020 a setembro de 2021. Dessa maneira, pode-se perceber que a busca por esse xarope é resultado da eficiência comprovada da redução dos efeitos voltados para a tosse, gripe, bronquite e problemas respiratórios como asma.

Com isso, sugere-se estudos mais aprofundados sobre efeitos do uso irracional ou automedicação desse medicamento em crianças devido à escassez de pesquisas e de literatura.

\section{Referências}

Amorim, L. Passareli; C. Marucci, M. C. (2020) Composição química, atividade biológica e segurança de uso de plantas do gênero Mikania. Revista Fitos. Rio de Janeiro, v. 14(1), p.490-491.

Araújo, R. G; Carolli, E. S. P; Alves, C. S. B. (2020) Análise dos perfis de segurança e eficácia e medicamentos fitoterápicos. Revista Eletrônica Gestão e Saúde. Volume I, n.1, ano 1.

Dantas, A. D. S. Estudo de toxicidade reprodutiva e genotoxicidade do óleo essencial de Origanum majorana. Tese de Doutorado Universidade Federal do Rio Grande do Sul.Porto Alegre. 2019. https://www.lume.ufrgs.br/handle/10183/202214.

Diniz, A. K. M. F; Jales, A. L; Oliveira, B.M; Paulino, D.A; Rennale, E; Melo, E.R.F; Morais, H.F.A; Medeiros, I.I.B; Azevedo, F.C.C; Azevedo, C.C.S; Marcelino, E.M; Santos, M.C.Q; Mariz, S.R; Araújo, C.R.F.(2020) - Manual sobre o uso de plantas medicinais do Nordeste para sintomas gripais e ansiedade em tempos de pandemia pela COVID 19. Revista Saúde \& Ciência, 9(1), janeiro.

Esteves, C. O; Rodrigues, R. M; Martins, A. L. D; Vieira, R. A; Barbosa, J. L; Vilela, J. B. F. (2020) - Medicamentos fitoterápicos: prevalência, vantagens e desvantagens de uso na prática clínica e perfil e avaliação dos usuários. Revista Med. Set-out, Revista Med. set-out, 99(5), p.463-72.

Evangelista, S. S; Sampaio, F. C; Parente, R. C; Bandeira, M. F. C. L. Fitoterápicos na odontologia: estudo etnobotânico na cidade de Manaus. Revista Brasileira Pla.Med. Campinas, 15(4), 2019.p.513-519.

Fernandes, K. N. (2020) Avaliação da qualidade de medicamentos fitoterápicos manipulados em Belo Horizonte (MG): Análise Orgânica e Inorgânica. Belo Horizonte. Análise orgânica e inorgânica, v.XVII, v.87.

Gomes, M. G; Pereira, B. B. (2019).Toxidade e uso racional. Editora FUCAMP.

Machado, V. F. L. S; Moraes .F. C; Lima, A. P. S. O; Sobral, L. R; Moreira, A. E. (2018) Algoritmo para o manejo da tosse e prescrição farmacêutica de xarope de guaco $10 \%$ mikania glomerata S. no Sistema Único de Saúde em Itapeva/SP. Revista Científica Eletrônica de Ciência Aplicada da Fait, n.1, maio.

Meotti, F.L.et al. (2020) Avaliação do uso de medicamentos fitoterápicos por acadêmicos de farmácia da Universidade Paranaense Unidade de Francisco

Beltrão - PR - Brasil. Reserch, Society and Development, 9(11),p. 1- 12.

Nascimento, M.A.P. (2020) Interação medicamentosa entre fitoterápicos oferecidos pelo Sistema Único de Saúde e medicamentos convencionais. https://www.monografias.ufop.br/handle/35400000/2693.

Pereira A. S. et al. (2018). Metodologia da pesquisa científica. [free e-book]. Santa Maria/RS. Ed. UAB/NTE/UFSM.

Ribeiro, L. H. L. (2019) Análise dos programas de plantas medicinais e fitoterápicos no Sistema Único de Saúde (SUS) sob a perspectiva territorial. Ciência \& 
Research, Society and Development, v. 10, n. 15, e395101522855, 2021

(CC BY 4.0) | ISSN 2525-3409 | DOI: http://dx.doi.org/10.33448/rsd-v10i15.22855

Saúde Coletiva, 24, p.343-344.

Ribeiro, L. H. L. (2019) Análise dos programas de plantas medicinais e fitoterápicos no Sistema Único de Saúde (SUS) sob a perspectiva territorial. Ciência \& Saúde Coletiva, v.24, n.5.

Santos, T.P. (2019) Análise de bulas e rótulos de medicamentos fitoterápicos à base de Guaco (Mikania glomerata Srengel) no Município Muritiba - BA. Recuperado

http://famamportal.com.br:8082/jspui/bitstream/123456789/1929/1/DEUS\%20É\%20FIEL\%20entregar\%20\%281\%29\%20CD\%20mily\%203.pdf.

Severino, A. J. (2018). Metodologia do trabalho cientifico. São Paulo: Cortez.

Silva F. C.; Ribeiro; A. B.; Ribeiro, P. R. S. (2019) Avaliação da qualidade de plantas medicinais comercializadas no município de Imperatriz, Maranhã; Scientia Plena. 13(2), p. 1-9.

Silva, N.L; Teixeira, A.B. (2019). Avaliação da atividade antimicrobiana da espécie vegetal Mikania glomerata sprengel Cultvada no Horto de Plantas Medicinais em uma faculdade de Fortaleza. Revista Diálogos Acadêmicos, 8(2), jan/jun.

Silva, V.C.M; Vilar, D.A; Vilar, M.A.S; Mélo, M.C.S. Rodrigues, P.L. (2021). Análise antimicrobiana do extrato de Mikania glomerata. Research, Society of Development, 10(7), p. 455-460.

Silva, M.A; Marcelino, E.M; Silva, A; Mariz, S.R. (2021) Evaluation of practices and knowledge about phytotherapy and self-medication among nursing graduates: a cross-section study. https://rsdjournal.org/index.php/rsd/article/view/18173

Teófilo, V.N; Ulmann, L.A.C. (2021). O Uso de Mikania Glomerata no tratamento alternativo para doenças respiratórias: revisão de literatura. Brazilian Journal of Development, Curitiba 7(6), jun.

Vilar, D.A; Vilar, M.A.S; Brandao, M.P; Anjos, C.J.F; Silva, A.Z. Plantas medicinais: um guia prático. https://repositorio.ifs.edu.br/biblioteca/handle/123456789/1104 